\section{Understanding why T-cell receptors remain single}

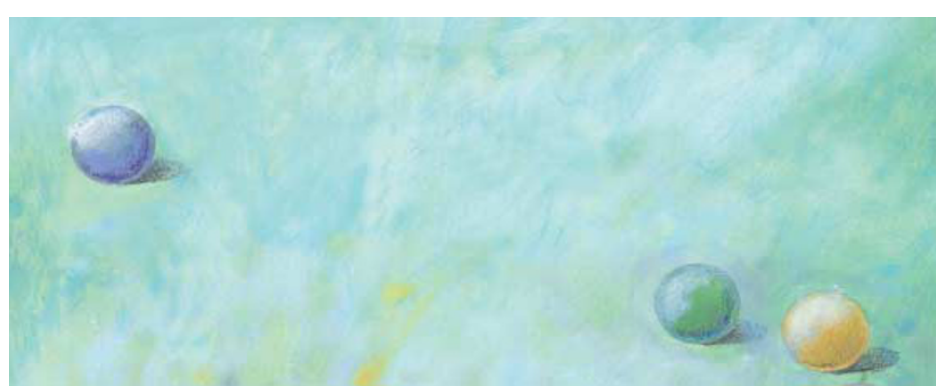

To ensure that $\mathrm{T}$ cells express a single T-cell receptor $\beta$-chain (TCR- $\beta$ ) that is, allelic exclusion - TCR- $\beta$ gene rearrangement is tightly regulated. Downregulation of V $\beta$ genesegment accessibility was thought to be crucial to the process of preventing secondary rearrangement after a functional TCR- $\beta$ has been generated. However, data published in Nature Immunology indicate that additional mechanisms regulate this process.

TCR- $\beta$ allelic exclusion is regulated at two stages of thymocyte development: at the double negative (DN) stage, when the TCR- $\beta$ alleles must rearrange asynchronously; and at the double positive (DP) stage — which is reached only after a maturation signal from the pre-TCR indicates successful TCR- $\beta$ gene rearrangement - when further TCR- $\beta$ gene rearrangement must be prevented. In this study, Jackson et al. set out to investigate the proposed role of $\mathrm{V} \beta$ gene-segment accessibility in the inhibition of Tcr- $\beta$ gene rearrangement after transition to the DP stage. They generated knock-in mice expressing a modified Tcr- $\beta$ allele in which the Tcr- $\alpha$ enhancer $\left(\mathrm{E}_{\alpha}\right)$ was positioned downstream of the V $\beta 12$ gene segment. Consistent with their hypothesis that the introduced $\mathrm{E}_{\alpha}\left(\mathrm{E}_{\alpha \mathrm{i}}\right)$ would maintain the $\mathrm{V} \beta$ chromatin in an active configuration in DP thymocytes, DP thymocytes from knock-in mice showed increased V $\beta$ germline transcription, histone modification and restriction-endonuclease accessibility compared with DP thymocytes from wild-type animals.
$\mathrm{V} \beta$-to-DJ $\beta$ rearrangement is downregulated in DP thymocytes from wild-type mice compared with rearrangement in DN thymocytes from the same animals. Surprisingly, despite the presence of $\mathrm{E}_{\alpha \mathrm{i}}$ increasing $\mathrm{V} \beta$ chromatin activation (and therefore accessibility) in DP thymocytes, $\mathrm{V} \beta$-to-DJ $\beta$ rearrangement in these cells was also downregulated comthymocytes.

Although inhibition of Tcr- $\beta$ gene rearrangement after transition to the $D P$ stage was not affected by the presence of $E_{\alpha i}, V \beta 12$ rearrangement was increased in DN thymocytes from with an increased proportion of peripheral $\mathrm{T}$ cells expressing both a TCR- $\beta$ containing V $\beta 12$ and a TCR- $\beta$ containing a distinct V $\beta$. Both $\beta$-chains were products of chromosomal rearrangement, indicating that the presence of $\mathrm{E}_{\alpha \mathrm{i}}$ disturbed allelic exclusion in DN thymocytes. This defect in allelic exclusion occurred despite the fact that the presence of $E_{\alpha i}$ did not alter $\mathrm{V} \beta$ gene-segment accessibility in DN thymocytes.

This study shows that factors other than chromatin accessibility regulate allelic exclusion in both the DN and the DP thymocyte compartments. Future studies will focus on identifying these additional controls at the molecular level.

Karen Honey

(D) References and links ORIGINAL RESEARCH PAPER Jackson, A., Kondilis, H. D., Khor, B., Sleckman, B. P. \& Krangel, M. S. Regulation of T cell receptor $\beta$ allelic exclusion at a level beyond accessibility. Nature Immunol. 6, 189-197 (2005). pared with rearrangement in DN $\mathrm{E}_{\alpha \mathrm{i}}$-knock-in mice. This correlated

\section{IN BRIEF}

\section{CHEMOKINES}

The role of CCL21 in recruitment of T-precursors to fetal thymi.

Liu, C. et al. Blood 105, 31-39 (2005).

In this study, Liu et al. developed a new in vitro time-lapse visualization technique to investigate the mechanisms that regulate the recruitment of T-cell precursors to the fetal thymus. Initial analysis showed that a proportion of fetal liver cells, blood cells or DN1 thymocytes were actively attracted towards an alymphoid fetal thymic lobe and that these cells were T-cell precursors. Recruitment of the T-cell precursors was mediated by MHC-class-II-expressing thymic epithelial cells and was abrogated in the presence of an inhibitor of G-protein-coupled receptors. Subsequently, the chemokines CCL21 and CCL25 were shown, using several assays, to contribute significantly to the active recruitment of T-cell precursors to the fetal thymus. Further studies are required to determine the molecular mechanism by which these chemokines mediate this effect.

\section{INNATE IMMUNITY}

\section{Drosophila peptidoglycan recognition protein LC} (PGRP-LC) acts as a signal-transducing innate immune receptor.

Choe, K.-M. et al. Proc. Natl Acad. Sci. USA 102, 1122-1126 (2005).

Drosophila melanogaster peptidoglycan-recognition protein LC (PGRP-LC) is a transmembrane protein that is required for antibacterial responses mediated by a signalling pathway that involves the death-domain protein IMD and the nuclear factor- $\kappa \mathrm{B}-$ family member Relish. Because the cytoplasmic domain of PGRP-LC has no sequence homology to characterized proteins, it is not known how it activates intracellular signalling. Using truncated PGRP-LC mutants, the authors show that its cytoplasmic domain binds IMD and is crucial for the induction of downstream signalling. The cytoplasmic domain also promotes dimerization of PGRP-LC, which might be required for its activation and might increase the diversity of ligand recognition through heterodimerization with other PGRP-LC isoforms.

\section{DENDRITIC CELLS}

Selective Rac1 inhibition in dendritic cells diminishes apoptotic cell uptake and cross-presentation in vivo.

Kerksiek, K. M. et al. Blood 105, 742-749 (2005).

Previous in vitro studies have indicated that the RHO guanosine triphosphatase RAC1 is important for endocytosis and crosspresentation by dendritic cells (DCs). In this paper, the authors confirm these results in vivo. Because knocking out RAC1 function in mice is embryonic lethal, they generated transgenic mice that express a DC-specific dominant-negative form of RAC1. Transgenic mice showed defective $\mathrm{CD} 8^{+} \mathrm{DC}$ development and function, and although DC migration was unaffected, apoptotic-cell uptake and cross-presentation by $\mathrm{CD}^{+}$DCs was severely impaired. This reduced their ability to induce bacteria-specific T-cell responses, indicating an important role for RAC1 in $\mathrm{CD}^{+} \mathrm{DC}$ function in vivo. 